\title{
Indicadores de qualidade em terapia nutricional: análise da evolução do serviço pós-
}

\section{implantação}

\author{
Quality indicators in nutritional therapy: analysis of the evolution of post-implantation service \\ Indicadores de calidad en terapia nutricional: análisis de la evolución del servicio post- \\ implantación
}

Recebido: 22/11/2021 | Revisado: 01/12/2021 | Aceito: 10/12/2021 | Publicado: 17/12/2021

Larissa Menezes Santos

ORCID: https://orcid.org/0000-0002-9026-141X Hospital Universitário de Sergipe, Brasil E-mail: nutlarissamenezes@gmail.com

Márcia Ferreira Cândido de Souza

ORCID: https://orcid.org/0000-0001-6812-2336 Hospital Universitário de Sergipe, Brasil E-mail: nutrimarciacandido@gmail.com

\begin{abstract}
Resumo
Introdução Os Indicadores de Qualidade em Terapia Nutricional (IQTN) são importantes ferramentas de avaliação e monitoramento de qualidade da Terapia Nutricional, uma vez que identificam possíveis dificuldades e falhas relacionadas aos cuidados nutricionais e possibilitam uma intervenção precoce. Objetivo Avaliar a implantação dos Indicadores de Qualidade em Terapia Nutricional no serviço de Terapia Nutricional do Hospital Universitário de Sergipe. Metodologia Estudo retrospectivo observacional realizado com dados sobre a pontuação diária dos Indicadores de Qualidade em Terapia Nutricional (IQTN) de pacientes adultos e idosos, de ambos os sexos, sob Terapia Nutricional Enteral (TNE) e/ou Terapia Nutricional Parenteral (TNP) por pelo menos 72 horas. Foram analisados doze IQTN em dois momentos: primeiro mês e 12 meses após a implantação. Utilizou-se as metas preconizadas pelo International Life Sciences Institute (ILSI) do Brasil e os momentos foram comparados pelo teste Wilcoxon com grau de significância de $\mathrm{p} \leq 0,05$. Resultados e discussão Foi demonstrado que dos 12 IQTN, 6 não atingiram as metas no momento inicial e a ultrapassaram ao final do estudo; 1 melhorou, apesar de permanecer abaixo da meta nos dois momentos $(\mathrm{p}=0,012)$; e os demais indicadores se mantiveram dentro das metas preconizadas durante todo o estudo. A significativa melhora na frequência dos indicadores demonstra a otimização da atenção nutricional e maior qualidade da assistência aos pacientes em Terapia Nutricional. Conclusão Foi demonstrado por meio da análise da implantação do IQTN que houve uma significativa evolução no serviço de terapia nutricional do hospital universitário avaliado.
\end{abstract}

Palavras-chave: Terapia nutricional; Indicadores; Gestão de qualidade.

\begin{abstract}
Introduction Quality Indicators in Nutrition Therapy (IQTN) are important tools for evaluating and monitoring the quality of Nutrition Therapy, as they identify possible difficulties and failures related to nutritional care and enable early intervention. Objective To evaluate the implementation of Quality Indicators in Nutritional Therapy in the Nutritional Therapy service of the University Hospital of Sergipe. Methodology Observational retrospective study carried out with data on the daily score of Quality Indicators in Nutritional Therapy (IQTN) of adult and elderly patients, of both sexes, undergoing Enteral Nutritional Therapy (ENT) and/or Parenteral Nutritional Therapy (NPT) for at least 72 hours. Twelve IQTN were analyzed in two moments: first month and 12 months after implantation. The goals recommended by the International Life Sciences Institute (ILSI) of Brazil were used and the moments were compared by the Wilcoxon test with a significance level of $\mathrm{p} \leq 0.05$. Results and discussion It was demonstrated that of the 12 IQTN, 6 did not reach the goals at the beginning and surpassed them at the end of the study; 1 improved, despite remaining below the target in both moments $(\mathrm{p}=0.012)$; and the other indicators remained within the recommended goals throughout the study. The significant improvement in the frequency of indicators demonstrates the optimization of nutritional care and better quality of care for patients in Nutritional Therapy. Conclusion It was demonstrated through the analysis of the implementation of the IQTN that there was a significant evolution in the nutritional therapy service of the evaluated university hospital.
\end{abstract}

Keywords: Nutritional therapy; Indicators; Quality management. 


\begin{abstract}
Resumen
Introducción Los Indicadores de Calidad en la Terapia Nutricional (IQTN) son herramientas importantes para evaluar y monitorear la calidad de la Terapia Nutricional, ya que identifican posibles dificultades y fallas relacionadas con la atención nutricional y permiten una intervención temprana. Objetivo Evaluar la implementación de Indicadores de Calidad en Terapia Nutricional en el servicio de Terapia Nutricional del Hospital Universitario de Sergipe. Metodología Estudio observacional retrospectivo realizado con datos sobre la puntuación diaria de Indicadores de Calidad en Terapia Nutricional (IQTN) de pacientes adultos y ancianos, de ambos sexos, sometidos a Terapia Nutricional Enteral (ORL) y / o Terapia Nutricional Parenteral (NPT) durante al menos 72 horas. Se analizaron doce IQTN en dos momentos: primer mes y 12 meses después del implante. Se utilizaron las metas recomendadas por el Instituto Internacional de Ciencias de la Vida (ILSI) de Brasil y los momentos se compararon mediante la prueba de Wilcoxon con un nivel de significancia de $\mathrm{p} \leq 0.05$. Resultados y discusión Se demostró que de las 12 IQTN, 6 no alcanzaron las metas al inicio y las superaron al final del estudio; 1 mejoró, a pesar de permanecer por debajo del objetivo en ambos momentos $(\mathrm{p}=0,012)$; y los demás indicadores se mantuvieron dentro de las metas recomendadas a lo largo del estudio. La mejora significativa en la frecuencia de los indicadores demuestra la optimización de la atención nutricional y una mejor calidad de la atención a los pacientes en Terapia Nutricional. Conclusión Se demostró a través del análisis de la implementación de la IQTN que hubo una evolución significativa en el servicio de terapia nutricional del hospital universitario evaluado.
\end{abstract}

Palabras clave: Terapia nutricional; Indicadores; Gestión de la calidad.

\title{
1. Introdução
}

A desnutrição hospitalar é comum e está frequentemente associada com um mau resultado clínico (Vargas et al, 2018), relacionando-se intimamente com um maior risco de complicações como infecções e lesões por pressão, prolongando tempo de permanência hospitalar (Gonçalves et al., 2017), aumentando o custo da hospitalização e o risco de mortalidade (Abbade, 2020). Pacientes desnutridos ou com risco de desnutrição, quando identificados precocemente, se beneficiam da Terapia Nutricional (Fontes et al., 2016).

A Terapia Nutricional é recomendada visando a prevenção da desnutrição, manutenção ou recuperação do estado nutricional (Guilherme, Silva, Casado e Burgos, 2020), por meio da nutrição parenteral e/ou enteral. Esta deve ser instituída e monitorada pelos profissionais que compõem a Equipe Multiprofissional de Terapia Nutricional - EMTN (Brasil, 1998). O acompanhamento desse processo é realizado através de informações que agregadas se transformam em dados, ou seja, indicadores (Guimarães, 2015).

Os indicadores de qualidade são critérios quantitativos utilizados para analisar a qualidade da atenção em saúde (Alves \& Borges, 2019). Os indicadores de qualidade em Terapia Nutricional (IQTN) são a ferramenta mais utilizada atualmente pela Equipe Multiprofissional de Terapia Nutricional (EMTN), para avaliação do cumprimento de metas relacionadas à evolução do estado nutricional e consequentemente da qualidade do serviço prestado (Van Aanholt \& Rey, 2015; Valois et al., 2018).

Os Indicadores de Qualidade em Terapia Nutricional (IQTN) são importantes ferramentas de avaliação e monitoramento de qualidade da Terapia Nutricional, uma vez que identifica possíveis dificuldades e falhas relacionadas aos cuidados nutricionais e possibilita uma intervenção precoce, melhorando a qualidade da assistência nutricional (Santana e Ceniccola, 2017).

Sendo assim, os IQTN surgem como uma nova perspectiva na avaliação da Terapia Nutricional e incorporação de gestão de qualidade na assistência aos pacientes em TN, de modo a garantir condutas assertivas e resultados favoráveis, com efetiva recuperação clínica a baixos custos (Hammes, 2019). Diante disso, o presente estudo teve como objetivo avaliar, por meio dos IQTN, a evolução do serviço de Terapia Nutricional do Hospital Universitário de Sergipe. 


\section{Metodologia}

Trata-se de um estudo retrospectivo observacional realizado com dados sobre a pontuação diária dos Indicadores de Qualidade em Terapia Nutricional (IQTN) coletados nos registros da Equipe Multiprofissional de Terapia Nutricional (EMTN) do Hospital Universitário, que incluem pacientes adultos e idosos de ambos os sexos sob Terapia Nutricional Enteral (TNE) e/ou Terapia Nutricional Parenteral (TNP) por pelo menos 72 horas.

Foram analisados os doze IQTN implantados na instituição: frequência de realização de triagem nutricional em até 48 horas da admissão; frequência de estimativa de gasto energético e necessidade proteica; frequência de pacientes que atingiram 80\% das necessidades em 72 horas; frequência de jejum digestório por mais de 24 horas; frequência de saída inadvertida de SNE; frequência de obstrução de SNE; frequência de diarreia; frequência de constipação; frequência de disfunção glicêmica; frequência de reavaliação periódica do planejamento nutricional; frequência de infecção de cateter venoso central em pacientes em TNP; frequência de flebite decorrente de cateter venoso periférico em pacientes em TNP (Santos, 2020).

O Quadro 1 apresenta os indicadores com suas respectivas fórmulas e metas preconizadas pelo International Life Sciences Institute (ILSI) do Brasil, que foram utilizadas como parâmetro nesse estudo.

Quadro 1. Indicadores de Qualidade em Terapia Nutricional, respectivas fórmulas e metas.

\begin{tabular}{|c|c|c|}
\hline Indicador de Qualidade & Fórmula & Meta \\
\hline $\begin{array}{l}\text { Frequência de realização de triagem nutricional em até } \\
48 \text { h da admissão (TNE) }\end{array}$ & $\frac{\mathrm{N}^{\mathrm{o}} \text { de triagens nutricionais em } 48 \mathrm{~h} \times 100}{\mathrm{~N}^{\mathrm{o}} \text { total de admissões em TNE em } 48 \mathrm{~h}}$ & $\geq 90 \%$ \\
\hline $\begin{array}{l}\text { Frequência de estimativa de gasto energético e } \\
\text { necessidade proteica (TNE) }\end{array}$ & $\frac{\mathrm{N}^{\mathrm{o}} \text { de pacientes em TNE com avaliação de gasto energético e proteico x } 100}{\mathrm{~N}^{\circ} \text { total de pacientes em TNE }}$ & $\geq 80 \%$ \\
\hline $\begin{array}{l}\text { Frequência de pacientes que atingiram } 80 \% \text { das } \\
\text { necessidades em } 72 \text { horas (TNE) }\end{array}$ & $\begin{array}{c}\frac{\mathrm{N}^{\circ} \text { de pacientes em TNE que atingiram } 80 \% \text { das necessidades em } 72 \mathrm{~h} \times 100}{\underline{\mathrm{N}^{\circ} \text { total de pacientes em TNE }}}\end{array}$ & $\geq 80 \%$ \\
\hline $\begin{array}{l}\text { Frequência de jejum digestório por mais de } 24 \text { horas } \\
\text { (TNE) }\end{array}$ & $\frac{\mathrm{N}^{\circ} \text { de pacientes em TNE em jejum maior que } 24 \mathrm{~h} \mathrm{x} 100}{\mathrm{~N}^{\circ} \text { total de pacientes em TNE }}$ & $\leq 10 \%$ \\
\hline Frequência de saída inadvertida de SNE (TNE) & $\frac{\mathrm{N}^{\circ} \text { de saída inadvertida de SNE x } 100}{\mathrm{~N}^{\mathrm{o}} \text { total de pacientes em TNE x } \mathrm{N}^{\mathrm{o}} \text { dias com SNE }}$ & $<5 \%$ \\
\hline Frequência de obstrução de SNE (TNE) & $\frac{\mathrm{N}^{\mathrm{o}} \text { de sondas obstruídas em pacientes em TNE x } 100}{\mathrm{~N}^{\mathrm{o}} \text { total de pacientes em TNE/dia }}$ & $\leq 5 \%$ \\
\hline Frequência de constipação (TNE) & $\frac{\mathrm{N}^{\mathrm{o}} \text { de pacientes em TNE com constipação x } 100}{\mathrm{~N}^{\circ} \text { total de pacientes em TNE }}$ & $\leq 20 \%$ \\
\hline Frequência de disfunção glicêmica (TNE) & $\frac{\mathrm{N}^{\circ} \text { de pacientes em TNE com hipo ou hiperglicemia x } 100}{\mathrm{~N}^{\circ} \text { total de pacientes em TNE }}$ & $<7 \%$ \\
\hline $\begin{array}{l}\text { Frequência de reavaliação periódica do planejamento } \\
\text { nutricional (TNE) }\end{array}$ & $\frac{\mathrm{N}^{\circ} \text { de pacientes em TNE sem reavaliação por mais de } 7 \text { dias x } 100}{\mathrm{~N}^{\mathrm{o}} \text { total de pacientes em TNE internados por mais de } 7 \text { dias }}$ & $>75 \%$ \\
\hline $\begin{array}{l}\text { Frequência de infecção de Cateter Venoso Central } \\
\text { (TNP) }\end{array}$ & $\frac{\mathrm{N}^{\mathrm{o}} \text { de infecções de CVC em paciente em TNP x } 100}{\mathrm{~N}^{\circ} \text { total de dias de CVC em pacientes em TNP }}$ & $<5 \%$ \\
\hline $\begin{array}{l}\text { Frequência de flebite decorrente de inserção de Cateter } \\
\text { Venoso Periférico (TNP) }\end{array}$ & $\frac{\mathrm{N}^{\mathrm{o}} \text { de infeccões de CVP em paciente em TNP x } 100}{\mathrm{~N}^{\circ} \text { total de dias de CVP em pacientes em TNP }}$ & $<5 \%$ \\
\hline
\end{tabular}

Fonte: ILSI Brasil (2018).

Os dados foram armazenados em planilha do Microsoft Excel e analisados em dois momentos: no primeiro mês (momento 1) e 12 meses após implantação (momento 2). Todas as análises foram realizadas em um programa estatístico. Para a estatística descritiva foram utilizadas as médias, desvios-padrões e frequências. Para a comparação dos dois momentos, utilizou-se o Teste de Wilcoxon com grau de significância de 5\%. 
O presente trabalho foi aprovado pelo Comitê de Ética e Pesquisa (CEP) da Universidade Federal de Sergipe, sob o número de parecer 4.083.265.

\section{Resultados e Discussão}

Foram analisados dados de registro de 58 pacientes com idade média de $63 \pm 15$ anos, sendo $63,8 \%$ (n=37) do gênero masculino. No que diz respeito ao tipo de Terapia Nutricional instituída, $81 \%(n=47)$ da amostra fez uso de Nutrição Enteral (NE) exclusiva, $10 \%$ (n=6) Nutrição Enteral associada a Nutrição Parenteral (NE + NPT) e 9\% (n=5) Nutrição Parenteral (NPT) exclusiva.

O primeiro momento da avaliação da implantação dos IQTN (momento 1) demonstrou que os indicadores denominados frequência de triagem nutricional em até 48 horas da admissão, frequência de estimativa de gasto energético e necessidade proteica, frequência de pacientes que atingiram $80 \%$ da meta em 72 horas e frequência de reavaliação periódica do planejamento nutricional apresentaram-se abaixo da meta. Os indicadores denominados frequência de disfunção glicêmica, frequência de constipação e frequência de jejum digestório por mais de 24 horas apresentaram-se acima da meta préestabelecida (Gráfico 1).

Gráfico 1. Comparativo entre os resultados do primeiro momento da implantação dos IQTN e a meta.

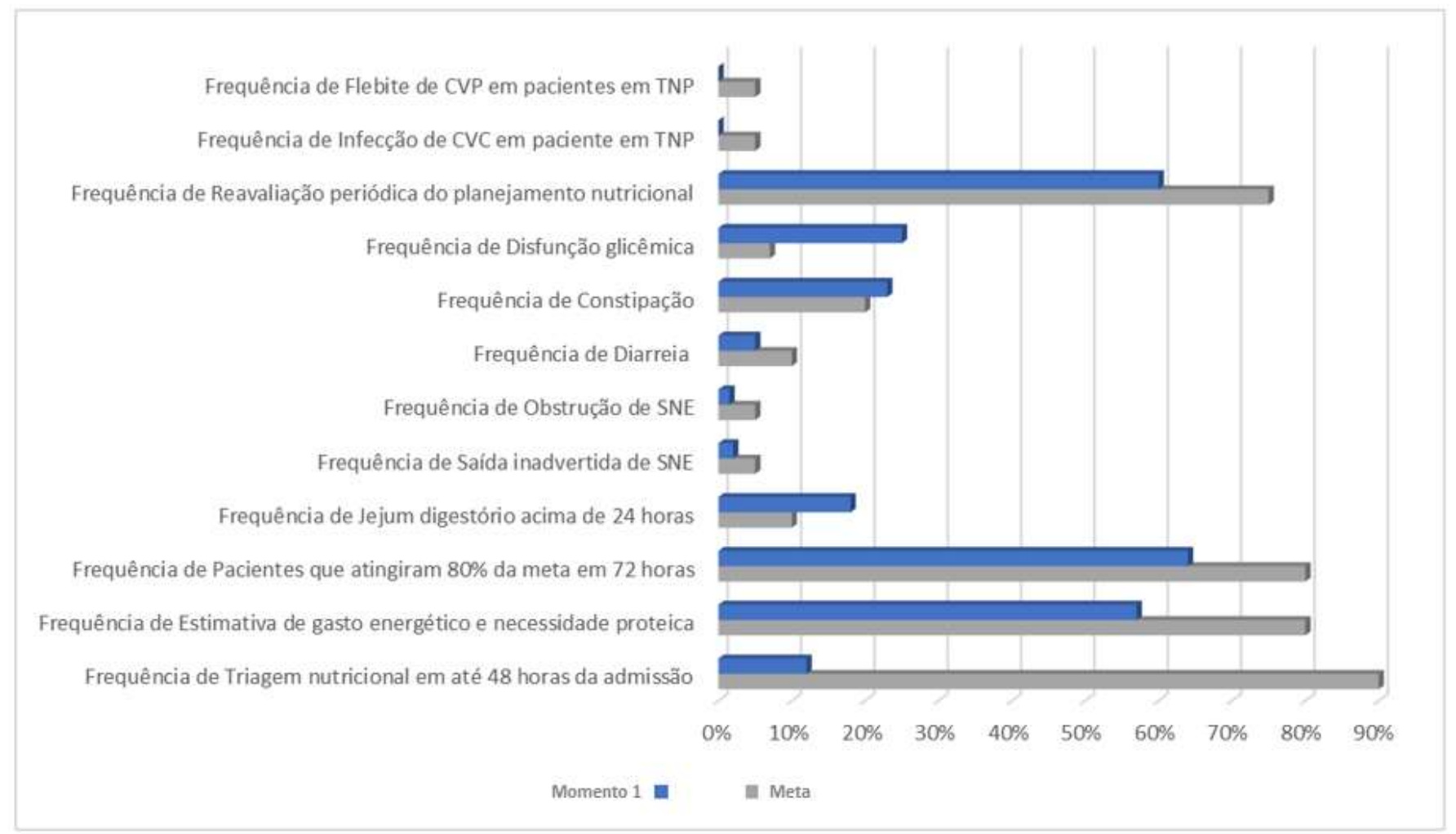

Legenda: TNE - Terapia Nutricional Enteral; SNE - Sonda Nasoenteral; CVC - Cateter Venoso Central; CVP -Cateter Venoso Periférico; TNP - Terapia Nutricional Parenteral. Fonte: Autores.

$\mathrm{Na}$ comparação do segundo momento (momento 2) com a meta, é possível visualizar que 4 indicadores anteriormente considerados abaixo da meta (indicadores de frequência de triagem nutricional em até 48 horas da admissão, estimativa de gasto energético e necessidade proteica, pacientes que atingiram $80 \%$ da meta em 72 horas e reavaliação periódica do planejamento nutricional) ultrapassaram esse parâmetro e 3 que apresentavam-se acima da meta (indicadores de frequência de disfunção glicêmica, constipação e jejum digestório por mais de 24 horas) foram reduzidos aos percentuais 
aceitáveis. Somente o indicador frequência de pacientes que atingiram $80 \%$ de meta em 72 horas esteve nos dois momentos abaixo da meta (Gráfico 2).

Gráfico 2. Comparativo entre os resultados do segundo momento da implantação dos IQTN e a meta.

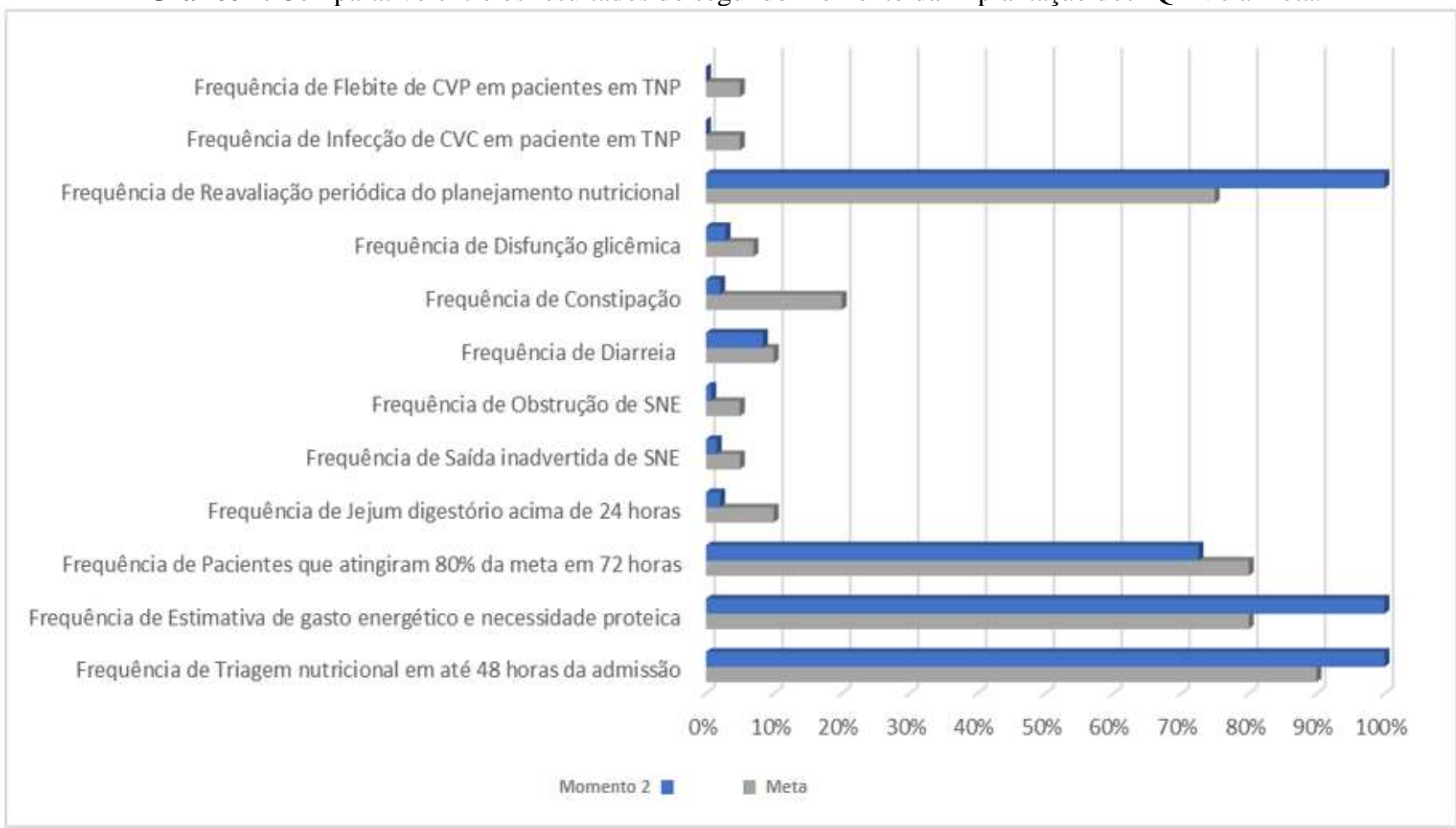

Legenda: TNE - Terapia Nutricional Enteral; SNE - Sonda Nasoenteral; CVC - Cateter Venoso Central; CVP -Cateter Venoso Periférico; TNP - Terapia Nutricional Parenteral. Fonte: Autores.

Quanto ao indicador denominado frequência de pacientes que atingiram 80\% das necessidades em 72 horas, apesar de não ter sido atingida a meta no segundo momento do estudo, houve uma significativa evolução $(\mathrm{p}=0,012)$ desse parâmetro, observando a melhora dos resultados quando comparado com o primeiro momento. Isso sugere uma adequação mais assertiva da Terapia Nutricional, o que contribui para manutenção ou melhora do estado nutricional e desfechos clínicos mais favoráveis.

Não obstante, é preciso contar com um esforço multidisciplinar, buscando uma prescrição dietética adequada, assim como a infusão da Nutrição Enteral em doses adequadas a fim de alcançar os benefícios que a mesma pode proporcionar. Tão importante quanto a prescrição adequada da TNE é a certeza de que o paciente estará recebendo a dieta prescrita (Mendonça \& Guedes, 2017).

Disfunção glicêmica, alterações no ritmo intestinal e jejum digestório prolongado - seja por instabilidade hemodinâmica ou para realização de exame e/ou procedimento - limitam a progressão da terapia nutricional (Santos et al, 2017). Portanto, a regularização dos indicadores frequência de disfunção glicêmica, constipação e jejum digestório por mais de 24 horas pode estar correlacionada à melhora da frequência de pacientes que atingiram $80 \%$ das necessidades em 72 horas.

A partir do comparativo dos resultados, primeiro e segundo momento após a implantação dos IQTN, foi possível observar uma evolução significativa na qualidade do serviço de terapia nutricional no hospital onde foi realizado o estudo (Tabela 1). Destaque para os indicadores frequência de triagem nutricional nas primeiras 48 horas, frequência de estimativa de gasto energético e necessidade proteica e frequência de reavaliação periódica do planejamento nutricional atingirem a frequência de $100 \%$ no segundo momento do estudo, sobretudo o de triagem nutricional por partir de uma frequência muito abaixo da meta (12\%). 
Tabela 1. Análise comparativa dos resultados dos dois momentos da implantação dos IQTN.

\begin{tabular}{|c|c|c|c|}
\hline Indicador de Qualidade em Terapia Nutricional (IQTN) & Momento $1^{1}(\%)$ & Momento $2^{2}(\%)$ & $\mathbf{p}^{*}$ \\
\hline Frequência de Triagem nutricional nas primeiras 48 horas da admissão & 12 & 100 & $\mathbf{0 , 0 1 1}$ \\
\hline Frequência de Estimativa de gasto energético e necessidade proteica & 57 & 100 & $\mathbf{0 , 0 1 1}$ \\
\hline Frequência de Pacientes que atingiram $80 \%$ da meta em 72 horas & 64 & 72,6 & 0,012 \\
\hline Frequência de Jejum digestório por mais de 24 horas & 18 & 2,1 & $\mathbf{0 , 0 1 2}$ \\
\hline Frequência de Saída inadvertida de SNE & 2 & 1,6 & 0,123 \\
\hline Frequência de Obstrução de SNE & 1,5 & 0,8 & $\mathbf{0 , 0 1 1}$ \\
\hline Frequência de Diarreia & 5 & 8,4 & 0,011 \\
\hline Frequência de Constipação & 23 & 2,1 & $\mathbf{0 , 0 1 2}$ \\
\hline Frequência de Disfunção glicêmica & 25 & 2,9 & $\mathbf{0 , 0 1 1}$ \\
\hline Frequência de Reavaliação periódica do planejamento nutricional & 60 & 100 & $\mathbf{0 , 0 1 0}$ \\
\hline Frequência de Infecção de CVC em paciente em TNP & 0 & 0 & 1,00 \\
\hline Frequência de Flebite de CVP em pacientes em TNP & 0 & 0 & 1,00 \\
\hline
\end{tabular}

Legenda: Momento $1^{1}$ - Primeiro mês da Implantação dos IQTN; Momento $2^{2}$ - Após 12 meses de implantação dos IQTN; \% - média percentual mensal; TNE - Terapia Nutricional Enteral; SNE - Sonda Nasoenteral; CVC - Cateter Venoso Central; CVP - Cateter Venoso Periférico; TNP - Terapia Nutricional Parenteral; *Teste de Wilcoxon. Fonte: Autores.

Foi realizado um estudo para identificar os 10 IQTN considerados mais úteis, práticos, de fácil execução e de baixo custo, além da sua importância no ambiente hospitalar. A lista desses indicadores foi denominada Top Ten e teve como primeiro indicador a avaliação do risco nutricional por meio da realização de triagem nutricional (Verotti, Torrinhas, Corona e Waitzberg, 2015), que permite identificar precocemente o risco de desnutrição e, a partir daí, direcionar melhores estratégias no suporte nutricional.

A European Society of Parenteral and Enteral Nutrition (ESPEN) recomenda que todos os indivíduos admitidos na unidade hospitalar devem ser submetidos à triagem nutricional para identificar se há risco nutricional. Caso seja identificado o risco, deve ser feita avaliação nutricional mais detalhada, e medidas deverão ser tomadas para monitorar, organizar e implementar a Terapia Nutricional (Brasil, 2016). Portanto, a substancial melhora desse indicador demonstra otimização da atenção nutricional aos pacientes em Terapia Nutricional.

A frequência de estimativa de gasto energético e necessidade proteica pode guiar a prescrição nutricional e prevenir complicações associadas à hiperalimentação e algumas importantes desordens metabólicas (Sá e Marshall, 2015). A oferta energético proteica aquém das necessidades também é uma problemática, uma vez que está associada ao risco de desnutrição, pior resposta imunológica, atraso no processo de cicatrização, aumento do risco de infecção, tempo de internamento e morbimortalidade (Toledo et al, 2018). Este indicador também está incluído na lista dos indicadores Top Ten (Verotti et al., 2015) e se completa com o indicador reavaliação periódica do planejamento nutricional, pela importância de se reavaliar essa oferta energética, uma vez que as necessidades variam com as oscilações clínicas e de tolerabilidade da terapia nutricional instituída e exigem monitoramento diário (Santos et al., 2017).

Positivamente, os indicadores denominados flebite de cateter venoso periférico e infecção de cateter venoso central em pacientes em Terapia Nutricional Parenteral se apresentaram dentro da meta nos dois momentos, uma vez que a contaminação do cateter venoso central é uma das mais temidas complicações da TNP (Castro et al., 2017).

Por fim, os resultados encontrados e avaliados a partir de metas recomendadas na literatura científica, evidenciam evolução na frequência dos indicadores analisados, podendo ser estimado que houve uma melhora da assistência nutricional aos pacientes em Terapia Nutricional. 


\section{Conclusão}

O presente estudo demonstrou por meio da avaliação após a implantação dos IQTN uma evolução significativa da qualidade da Terapia Nutricional no hospital onde foi realizada a pesquisa. Ademais, estudos mais amplos que consigam viabilizar a avaliação de cada IQTN distribuído pelos diversos setores hospitalares são sugeridos.

\section{Referências}

Abbade, E. B. (2020). Adoção de terapias nutricionais enteral e parenteral associada à redução da taxa de óbitos de pacientes neoplásicos. Medicina, 53(2):115-125

Alves, A. H. R., \& Borges, S. (2019). Indicadores de qualidade em terapia enteral: avaliação da assistência nutricional ao paciente hospitalizado. Braspen J, 34(1): 77-82.

Brasil. Ministério da Saúde (2016). Manual de terapia nutricional na atenção especializada hospitalar no âmbito do Sistema Único de Saúde - SUS. BrasíliaDF: Ministério da Saúde

Castro, M. G., Figueiredo, E. J. A., \& Matos, L. B. N. (2017). Equipe multiprofissional de terapia nutricional (pp. 159-168). Editora Atheneu.

Fontes, S. R., Henriques, G. S., Nahim-Safadi, C. M. A., Souza, A. S. B., \& Jansen, A. K. (2016). Triagem nutricional como ferramenta de organização da atenção nutricional hospitalar. Rev Bras Nutr Clin, 31(2): 124-8.

Gonçalves, C. V., Borges, L. R., Orlandi, S. P., \& Bertacco, R. T. A. (2017). Monitoramento de terapia nutricional enteral em unidade de terapia intensiva: adequação calórico-proteica e sobrevida. Braspen J, 32(4): 341-6.

Guimarães, M. P. (2015). Terapia nutricional: aspectos de qualidade e gerenciamento de riscos (pp. 39-44) Editora Atheneu.

Guilherme, L. G., Silva, L. L.B., Casado, A. H. S., \& Burgos, M. G. P. A. (2019). Terapia nutricional em pacientes oncológicos: realidade de um hospital de referência em Pernambuco. Nutr Clín Diet Hosp, 40(1):33-39.

Hammes, T. O. (2019). Indicadores de qualidade em terapia nutricional: uma revisão integrativa. Rev. Adm. Saúde. 19(77):e190.

ILSI (2018). Indicadores de qualidade em terapia nutricional: 10 anos de IQTN no Brasil: resultados, desafios e propostas (3a. ed.). ILSI Brasil.

Mendonça, M. R., \& Guedes, G. (2018). Terapia nutricional enteral em uma unidade de terapia intensiva: prescrição versus infusão. Braspen J, $33(1)$ : 54-57.

Sá, J. S. M., \& Marshall, N. G. (2015). Indicadores de qualidade em terapia nutricional como ferramenta de monitoramento da assistência nutricional no paciente cirúrgico. Rev Bras Nutr Clin, 30(2):100-5.

Santana, L. S., \& Ceniccola, G. D. (2017). Classificação de indicadores de qualidade em ouro e prata por cenário clínico do serviço público de acordo com especialistas em terapia nutricional. Braspen J, 32(4):369-74.

Santos, L. M. (2020). Protocolo Indicadores de Qualidade da Terapia Nutricional, de 17 de agosto de $2020 . \quad<$ http://siga.hu-ufs.ebserh/wpcontent/uploads/2016/02/PRT.EMTN_.003__INDICADORES_DE_QUALIDADE_EM_TERAPIA_NUTRICIONAL1.pdf>.

Santos, C. A., Firmino, H. H., Esmeraldo, M. L. F., Alfenas, R. C. G., Rosa, C. O. B., Ribeiro, A. Q., Almeida, L. F., \& Amorim, G. P. (2017). Perfil nutricional e fatores associados à desnutrição e ao óbito em pacientes com indicação de terapia nutricional. Braspen J, 32(1): 30-5.

Santos, G. F. C. G., Diament, D, Matsuba, C. S. T. \& Piovacari, S. M. F. (2017). Equipe multiprofissional de terapia nutricional (pp. 105-115). Editora Atheneu.

Toledo, D. O., Piovacari, S. M. F., Horie, L. M., Matos, L. B. N., Castro, M. G., Ceniccola, G. D., Corrêa, F. G., Giacomassi, I. W. S., Barrére, A. P. N., Campos, L. F., Verotti, C. C. G., Matsuba, C. S. T., Gonçalves, R. C., Falcão, H, Dib, R, Lima, T. E. C., Souza, I. A. O., Gonzalez, M. C., \& Correia, M. I. D. (2018). Campanha "Diga não à desnutrição": 11 passos importantes para combater a desnutrição hospitalar. Braspen J, 33(1): 86-100.

Valois, J. L. T. B., Soares, L. A., Feitosa, M. E. S., Holzbach, L. C., \& Pereira, R. J. (2018). Indicadores de qualidade em terapia nutricional em uma unidade de terapia intensiva neonatal de Palmas-TO. Revista Desafios, 5(3).

Van Aanholt, D. P. J., \& Rey, J. S. F. (2015). Terapia nutricional: aspectos de qualidade e gerenciamento de riscos (pp. 141-148). Editora Atheneu.

Vargas, P. M., Pretto, A. D. B., Massaut, K. B., Silva, E. P., Rodrigues, R. R., Marques, G. A. \& Moreira, A. N. (2018). Avaliação do estado nutricional de pacientes em uso de terapia nutricional enteral. Revista Brasileira de Obesidade, Nutrição e Emagrecimento, 12(75): 830-840.

Verotti, C. C. G., Torrinhas, R. S. M. M., Corona, L. P., \& Waitzberg, D. L. (2015). Design of quality indicators for oral nutritional therapy. Nutr Hosp, 31(6):2692-5. 